\title{
Taming the HIV/AIDS monster in our lifetime?
}

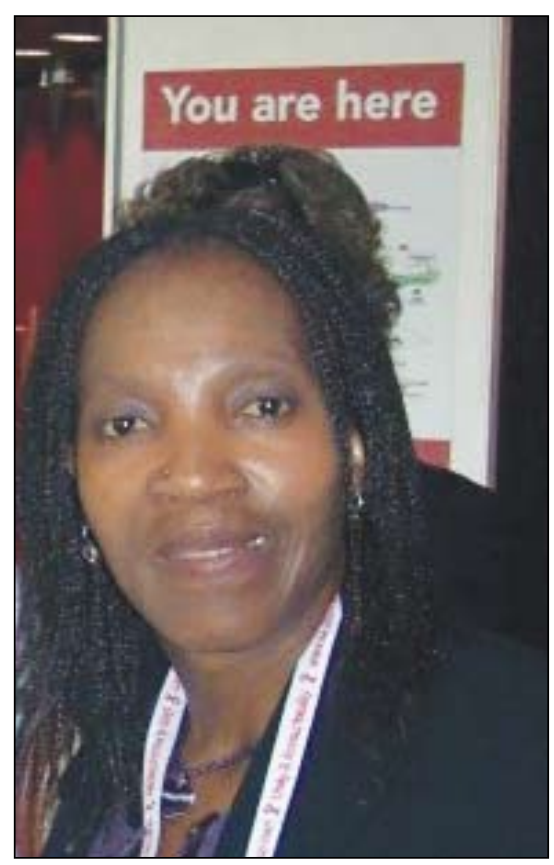

Dr Nono Simelela, CEO of the South African National AIDS Council.

She could have said that sufficient counselling rooms and trained staff exist, that the health system is 'patient friendly' enough to ensure most of the 1.65 million people who'll test HIV positive in the massive new campaign will be treated and retained.

\section{'Ramping up from}

430 exclusively "HIVaccredited" facilities to the 4300 existing State health care facilities is a huge, huge task. I can't deny that there'll be bottlenecks. It's not just commodities, it's human resources, storage challenges ... but for me, mainstreaming HIV care where you get everybody involved is the big win,' Simelela says.

But Dr Nono Simelela, CEO of the South African National AIDS Council (SANAC), has lived through an era of political make-believe and HIV denialism as the country's reluctant former AIDS chief. She knows the harsh reality of public health care delivery and some of the political arrogance that contributed to today's shameful outcomes.
In the new era of HIV pragmatism

Simelela is forthright about the challenges inherent in what is the most ambitious health care testing and counselling campaign involving HIV the world has seen. Testing 15 million of South Africa's 48 million people by June next year - and then treating those found positive - is no small ask of an overburdened public health care system that easily tips into dysfunction, prolonging suffering and costing lives.

The success of the campaign hinges on the long overdue integration of HIV/AIDS care into mainstream primary health care, major financial and logistical private and NGO health care sector involvement, massive government financial backing and creative human resources recruitment.

'Ramping up from 430 exclusively "HIV-accredited" facilities to the 4300 existing State health care facilities is a huge, huge task. I can't deny that there'll be bottlenecks. It's not just commodities, it's human resources, storage challenges ... but for me, mainstreaming HIV care where you get everybody involved is the big win,' Simelela says.

The lack of proper monitoring and evaluation means that nobody actually knows how the estimated 900000 people already on antiretroviral treatment (ART) are actually doing, how much slack the 2000 campaign volunteers so far (mainly retired nurses on fast-track up-skilling) will take up in the understaffed system, and what the actual counselling room availability is.

The upside, says Simelela, is that the budgeting for those who will need ART has been secured with R5 billion from treasury over the next 3 years (a 33\% hike in the ARV budget), plus R840 million pledged by PEPFAR over the remainder of this year and next year. The South African private sector has also come to the party with R706 million pledged so far while two major pharmacy chains are offering free health testing for the uninsured.

'Yes, the system is not as strong as we'd like it and there will be challenges, but at least in terms of the drug (ARVs) we can carry that. Like any other expansion, there will be those who have not initiated treatment even though they qualify, and that will be a challenge,' Simelela admitted.

\begin{tabular}{c}
\hline 'We either do this or \\
we keep going down. \\
My confidence is based \\
on us targeting TB/HIV \\
co-infection and pregnant \\
women in the campaign,' \\
Simelela adds.
\end{tabular}

\section{Children and pregnant women finally prioritised}

Besides unprecedented funding, she's also pinning her hopes on the new 1 April HIV treatment guidelines that upgrade and bring prophylactic intervention (triple therapy) for pregnant women with CD4 counts below 350 forward from 28 weeks to 14 weeks - and ART regardless of CD4 counts for all children under 1 year old. (Best practice prevention of mother-tochild transmission (PMTCT) continues.) Simelela believes that, taken together, these new measures will bring South Africa's under- 1 and under- 5 child mortality figures down from being the worst in the world and cut MTCT mortality from the current $15 \%$ to well under $5 \%$.

'We either do this or we keep going down. My confidence is based on us targeting TB/HIV co-infection and pregnant women in the campaign,' Simelela adds.

By South Africa's own (conservative) estimate 5.5 million South Africans are known to be HIV positive; by June next year this will have shot up to at least 7 million.

For the first time in years, there's a creative response to health care's human resource crisis. It's a simple initiative; scouring Health Professions Council of South Africa (HPCSA)'s member lists for potential retired or inactive volunteers plus a broad appeal for professionals to call in on Life Line's AIDS counselling line and offer their 
services. The HPCSA has waived registration fees for volunteers for the duration of their service only (to avoid potential moonlighting in future).

Simelela added that several large PEPFAR-funded NGOs, such as Right to Care, the Foundation for Professional Development and BroadReach, were helping provinces with the expansion by using their own trained people or by training others.

Celicia Serenata, SANAC's manager for resource tracking and donor co-ordination, told Izindaba that of the 4000 health care professionals who initially expressed interest, 'the last time I looked we had 2000 solid volunteers. Mainly nurses, but also social workers, psychologists and pharmacists.' There were very few doctors because, as Simelela explains, 'they'd have insufficient time to counsel and would be overwhelmed'. She urged private doctors who were 'not comfortable with volunteering' to refer patients to the nearest clinic.

\section{Laboratories will cope - SANAC}

The SANAC duo was confident that the National Health Laboratory Service (NHLS), which had expanded its services and was using couriers to pick up blood specimens country-wide, would cope.

They emphasised that the campaign was for comprehensive health care and testing, not just HIV / AIDS. Screening will include blood glucose and haemoglobin, and blood pressures will be taken while TB checks will consist of asking the 5 basic diagnostic indicator questions.

Clicks and Link pharmacies will offer free testing and counselling for all but haemoglobin levels to anyone who does not have medical health insurance, using counsellors up-skilled to the new State voluntary counselling and testing (VCT) protocols. Of major significance is that these protocols now include proactively offering an HIV test (so-called 'opt-out' testing), instead of waiting for patients to ask for one ('opt in') - State practice until early this year.

Among the members of the South African Business Coalition Against HIV / AIDS (SABCOHA) to contribute were Discovery Health (R5 million for equipment and training), Life Healthcare Hospitals (R500 000 for training), MedScheme (R2.5 million for 10 million condoms, R1 million by Aspen Pharmaceuticals for HIV test kits, and Roche Pharmaceuticals donating 4000 glucometers worth about R500 000.

Simelela said a major community awareness and social mobilisation campaign would prepare each of the first 9 focus districts ( 1 per province) in advance of a 2-month testing campaign, moving progressively in time across the country's 52 health districts.

Serenata explained that from the beginning of April another 513 facilities had been added to the 430 already initiating ART, with similar numbers being added every quarter of the financial year, but stressed that all 4300 facilities were now capable of doing VCT.

She warned, 'We'll probably start with the low hanging fruit (facilities best prepared for patient ART initiation) and it may well be that there are progressively less that come on stream every quarter'.

'I think he's testing the
system to see what it can
and cannot do. Linking HIV
to TB and other screening
programmes is his way of
seeing what we can do with
the resources we have.

\section{HIV Clinicians Society laud 'the new creativity'}

Dr Francois Venter, President of the Southern African HIV Clinicians Society, lavished praise on the new health minister, Dr Aaron Motsoaledi, for driving the new multi-pronged strategy.

'I think he's testing the system to see what it can and cannot do. Linking HIV to $\mathrm{TB}$ and other screening programmes is his way of seeing what we can do with the resources we have. If it was simple testing, it would be a wasted opportunity.'

In the last 3 - 4 years South Africa had escalated HIV testing dramatically with about 1.2 million people now having started therapy and some $200000+$ lost to follow-up. Most of the data suggested that these $15-20 \%$ of

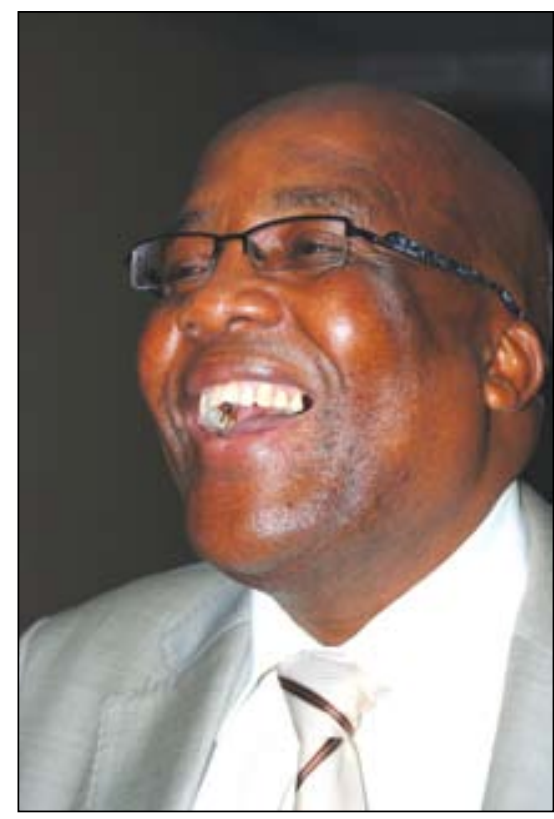

National Minister of Health, Dr Aaron Motsoaledi.

Picture: Chris Bateman

treatment 'initiates' were lost to followup (death, discontinued therapy, or reasons unknown) at 2 years, depending on the location. Venter said that while the data were robust and came from a large number of sentinel sites, in many outlying areas nobody had 'bothered to count'. 'We're not certain that everyone's doing OK because we concentrated on premier sites and quality checking systems are not in place,' he explained.

Under Dr Manto TshabalalaMsimang's health care reign 'lip service' was paid to primary health care and HIV but with a dire lack of intellectual resources. The most fundamental 'miss' was the lack of a human resources plan.

He was confident that Motsoaledi was aware that patients 'voted with their feet', avoiding long queues, rude staff, needless bureaucracy and drug stock outs and that late presentation for treatment was a function of these systemic weaknesses.

'He's already said that we're not using our existing health care staff properly. As for the current staffing boost - I didn't think we'd see this creativity in our lifetime, it really does feel like we're in a new country,' Venter enthused.

\section{Chris Bateman}

